\title{
Effects of Temperature and Pressure on the Micro-Brownian Motion in Poly(vinyl chloride)
}

\author{
Hiroyuki SASABE and Shogo SAIto \\ Electrotechnicl Laboratory, Tanashi, Tokyo, Japan.
}

(Received February 3, 1972)

\begin{abstract}
The rate of the micro-Browian motion of amorphous chain segments in poly(vinyl chloride) and the criterion for the glass transition were discussed from thermodynamic aspects. The procedure of calculation of excess entropy $S_{\mathrm{e}}$, excess enthalpy $H_{\mathrm{e}}$, and excess volume fraction $f$ from observable quantities is also described.

The rate of segmental motion was represented by the dielectric relaxation time for the $\alpha_{\text {a }}$ process, and measured as a function of temperature and pressure. It is concluded that the relaxation time is strongly dependent on $S_{\mathrm{e}}$ and/or $H_{\mathrm{e}}$ rather than on $f$, and that the glass transition is also determined by $S_{\mathrm{e}}$ and/or $H_{\mathrm{e}}$ rather than by $f$.

KEY WORDS Micro-Brownian Motion / Relaxation Time / Glass

Transition / Excess Volume / Excess Entropy / Excess Enthaly /
\end{abstract}

As a criterion for the glass transition, the free volume concept has been dominant; that is, a polymer is considered to vitrify when its fractional free volume is reduced to a critical value in the process of cooling at atmospheric pressure. If the free volume concept is also valid for vitrification procedure by application of a high hydrostatic pressure, it is expected that no change in net density should be observed while subjecting a polymer to a heating-compression-cooling-depressurization cycle. However the experimental results by Shishkin ${ }^{1}$ are inconsistent with this prediction; a residual density increase is confirmed. Thus the applicability of the free volume concept may become questionable at high pressure. According to Goldstein ${ }^{2}$ configurational entropy and configurational enthalpy must be taken into consideration as a possible criterion for the glass transition. Thus it is necessary to determine what factors are in control in the glass transition.

A similar question to that of the glass transition is raised in connection with the rate of the micro-Brownian motion of amorphous polymer chains. The rate is inversely proportional to the mechanical or the dielectric relaxation time $\tau$ for the so-called $\alpha_{\mathrm{a}}$ relaxation process. The temperature dependence of $\tau$ at $1 \mathrm{~atm}$. is successfully formulated by the Williams-Landel -Ferry (WLF) equation, ${ }^{3}$

$$
\log \frac{\tau(T)}{\tau\left(T_{\mathrm{g}}\right)}=-\frac{C_{1}\left(T-T_{\mathrm{g}}\right)}{C_{2}+\left(T-T_{\mathrm{g}}\right)}
$$

The pressure dependence of $\tau$ at fixed temperature is given by the Ferry-Stratton (FS) equation, ${ }^{4}$

$$
\log \frac{\tau(P)}{\tau(0)}=\frac{b_{1} P}{b_{2}-P}
$$

These equations can be derived not only from a concept of free volume but also from that of configurational entropy or configurational enthalpy. Parameters $C$ and $b$ can be represented as a function of any of these variables. Then one has to inquire which concept gives values of the parameters $C$ and $b$ that are consistent with the experimental data. It is the purpose of this paper to find out appropriate answers to these two questions.

In the earlier work, ${ }^{5}$ we have studied the temperature and pressure dependences of the relaxation time for the $\alpha_{\mathrm{a}}$ process and concluded that the free volume concept is valid for their description if the free volume is regarded as the excess volume, and that for most polymer samples $\log \tau$ changes linearly with pressure. However from the present work performed over wide ranges of temperature and pressure these conclusions should be considerably modified as follows: temperature and pressure dependences 
of the relaxation time for the $\alpha_{\mathrm{a}}$ process are expressed by the WLF and the FS equation, respectively, and are determined with excess entropy or excess enthalpy rather than with excess volume. We will show the grounds for this conclusion in the following sections.

\section{EXPERIMENTAL}

Dielectric constant $\varepsilon^{\prime}$ and dielectric loss $\varepsilon^{\prime \prime}$ for poly(vinyl chloride) (PVC) were measured as a function of frequency, temperature, and pressure. The average dielectric relaxation time $\tau(T, P)$ is defined as $\tau=1 / 2 \pi f_{\mathrm{m}}$, where $f_{\mathrm{m}}$ is the frequency corresponding to the maximum of the isothermal and isobaric absorption curve $\left(\varepsilon^{\prime \prime} v s\right.$. $\log f$ plot). Dielectric measurements were carried out over the wide ranges of frequency $\left(10^{-4} \sim\right.$ $\left.3 \times 10^{5} \mathrm{~Hz}\right)$, temperature $\left(20 \sim 126^{\circ} \mathrm{C}\right)$, and pressure $(1 \sim 3000 \mathrm{~atm}$.$) . Pressure and temperature$ dependences of d.c. conductivity for the sample were also studied in order to determine the glass transition point. Devices for the dielectric and d.c. conduction measurements at atmospheric and high pressures are described in a previous paper. $^{5}$

Figure 1 shows the effect of pressure on the logarithm of the relaxation time at various fixed temperatures for PVC. The relaxation time increases with increase in pressure, following the FS equation (eq 2).

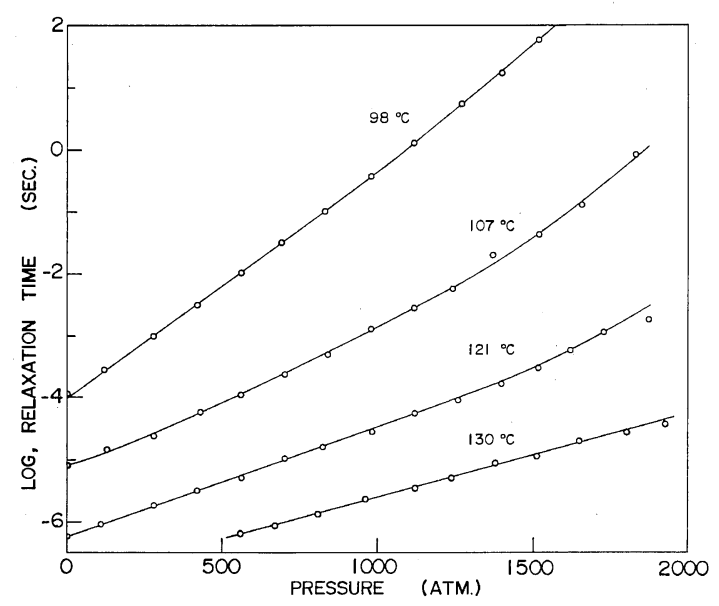

Figure 1. Effects of pressure on the relaxation time at various fixed temperatures.

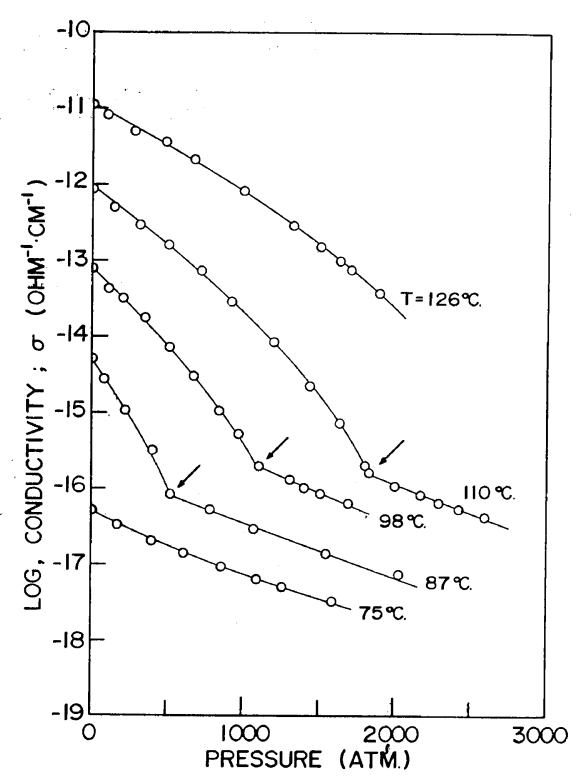

Figure 2. Isothermal plots of d.c. conductivity against pressure. Arrows indicate the glass-transition pressure $\boldsymbol{P}_{\mathrm{g}}$ at the temperatures.

Figure 2 shows the isothermal plots of d.c. conductivity against pressure for PVC. A break point on the isothermal curve corresponds to the glass-transition point, which will be discussed later.

\section{DISCUSSION}

\section{Factors Controlling Relaxation Time}

Definition of the excess entropy $\Delta S_{\mathrm{e}}$, the excess enthalpy $\Delta H_{\mathrm{e}}$, and the excess volume fraction $\Delta f$ and the procedure for their calculation from experimentally observable quantities are cited in the Appendix. The relations between $\log \tau$ and $\Delta S_{\mathrm{e}}, \Delta H_{\mathrm{e}}$, and $\Delta f$ at observed $(T, P)$ conditions can be obtained with the aid of Figures A1 A3. Results are shown in Figures 3 5, respectively, as a function of temperature and pressure.

If $\tau$ is controlled by only one variable, changes of $\tau$ with $T$ and $P$ can be estimated from $T$ and $P$ dependences of the variable. ${ }^{4}$ And if we choose the most suitable variable, the plots of $\tau$ vs. the variable should lie on a single curve even when $T$ and $P$ are both changed. As seen from Figure 5, isothermal curves (broken lines) are never reduced to the isobaric curve 


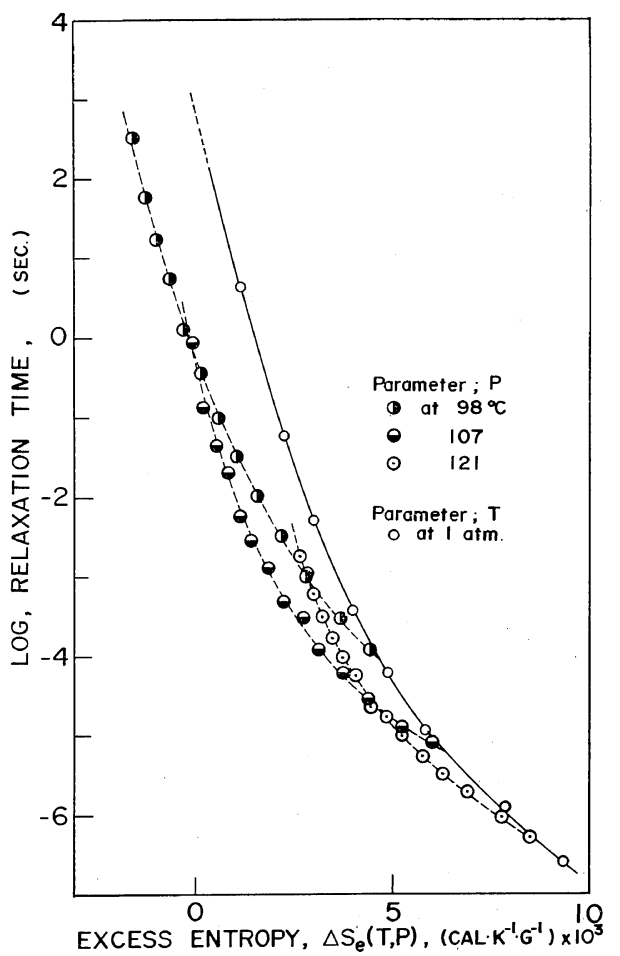

Figure 3. Relationship between relaxation time and excess entropy.

(solid line) perfectly. Deviation of the isothermal curves from the isobaric curve becomes remarkable with increases in the fixed temperature and the external pressure. Therefore the expression that $f$ determines the rate of segmental motion would be inadequate. On the other hand, isothermal curves (broken lines) at various fixed temperatures in Figures 3 and 4 are located closer to the isobaric curves (solid lines) in comparison with those in Figure 5. This result suggests that $\tau(T, P)$ is controlled by $S_{\mathrm{e}}$ and/or $H_{\mathrm{e}}$ rather than by $f$. This is in close agreement with the result obtained by Goldstein. ${ }^{2}$ It should be stressed, however, that both WLFand FS-type equations can be derived from the Adam-Gibbs theory based on the configurational entropy concept and that $S_{\mathrm{e}}$ is closely concerned with $f$ through the introduction of "vacant site."

\section{Application of the Adam-Gibbs Theory ${ }^{6}$}

The conclusion that the rate of the microBrownian motion of amorphous chain segments

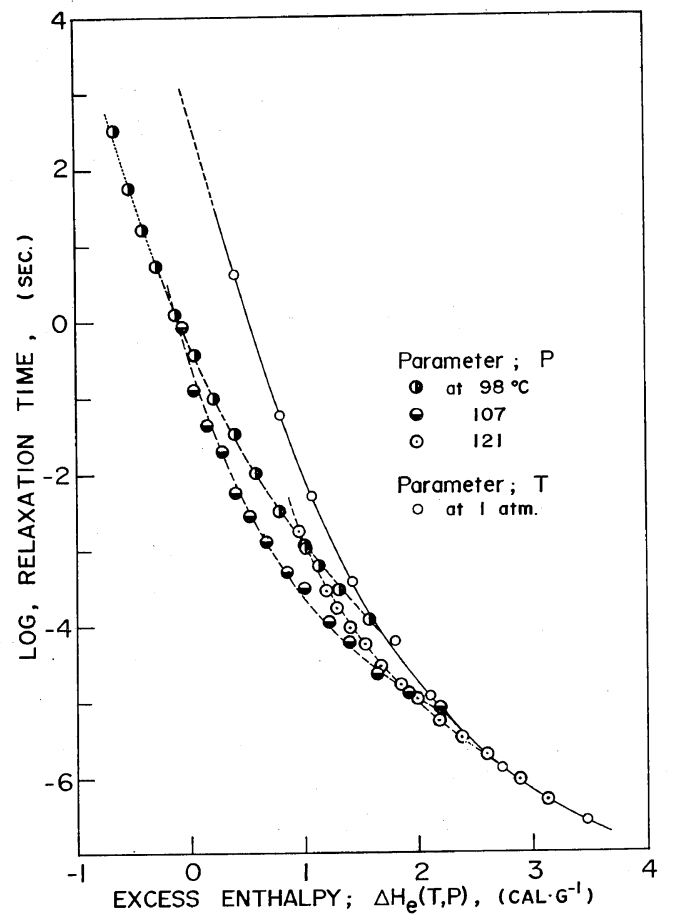

Figure 4. Relationship between relaxation time and excess enthalpy.

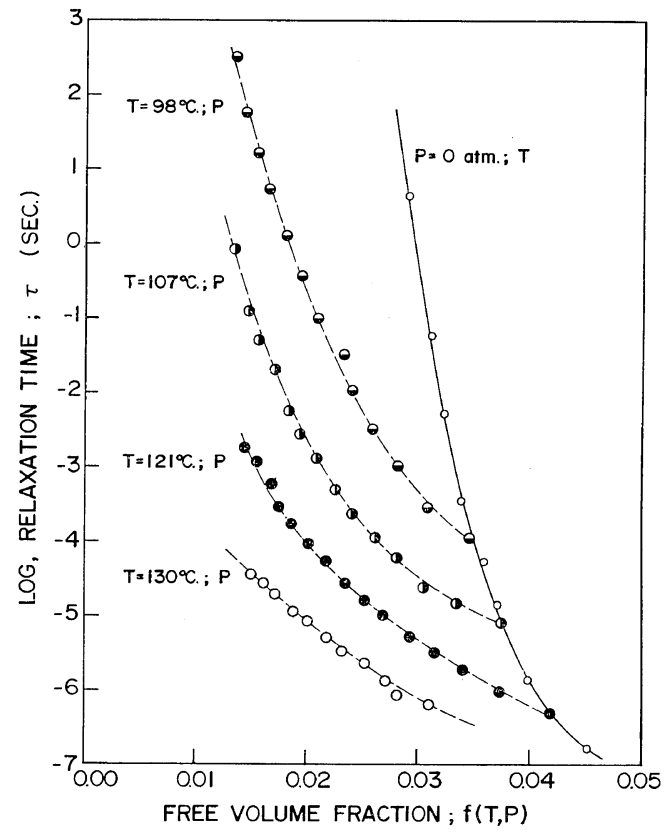

Figure 5. Relationship between relaxation time and excess volume. 
would be determined by $S_{\mathrm{e}}$ makes it possible to apply the Adam-Gibbs theory to the calculation of $S_{\mathrm{e}}\left(\right.$ not $\left.\Delta S_{\mathrm{e}}\right)$ at an arbitrary temperature and pressure. The Adam-Gibbs theory is a quantitative theory relating a configurational relaxation time and a configurational (excess) entropy, and gives the following relation

$$
\log \left[\tau(T) / \tau\left(T_{\mathrm{g}}{ }^{0}\right)\right]=-a_{1}\left(T-T_{\mathrm{g}}{ }^{0}\right) /\left[a_{2}+\left(T-T_{\mathrm{g}}{ }^{0}\right)\right]
$$

where

$$
\begin{aligned}
& a_{1}=C /\left[2.303 \Delta C_{\mathrm{P}} T_{\mathrm{g}}{ }^{0} \ln \left(T_{\mathrm{g}}{ }^{0} / T_{2}\right)\right], \\
& a_{2}=T_{\mathrm{g}}{ }^{0} \ln \left(T_{\mathrm{g}}{ }^{0} / T_{2}\right) /\left[1+\ln \left(T_{\mathrm{g}}{ }^{0} / T_{2}\right)\right],
\end{aligned}
$$

$C=\Delta \mu s_{\mathrm{c}}{ }^{*} / k, \Delta \mu$ is the potential energy hindering the cooperative rearrangement per monomer segment, $s_{\mathrm{c}}{ }^{*}$ the critical configurational entropy of a rearrangeable region, $T_{2}$ the second-order transition temperature where the excess entropy vanishes, and $k$ the Boltzmann constant. Equation 3 is similar in form to the WLF equation and parameters $a_{1}$ and $a_{2}$ correspond respectively to WLF parameters $C_{1}$ and $C_{2}$. Parameters $a_{1}$ and $a_{2}$ for the present PVC sample were calculated from the temperature dependence of the average relaxation time at $1 \mathrm{~atm}$., as listed in Table I. Table $\mathrm{I}$ also contains $T_{2}$ value for

Table I. Characteristic values in the PVC sample

\begin{tabular}{ll}
\hline$T_{\mathrm{g}^{0}}$, & $75^{\circ} \mathrm{C}$ \\
$C_{1}, a_{1}$, & 16.40 \\
$C_{2}, a_{2}$, & $35.71 \mathrm{deg}$ \\
$\tau \mathrm{g}^{0}$, & $10^{2.5} \mathrm{sec}$ \\
$\Delta C_{P}$, & $0.068 \mathrm{cal} \mathrm{g}^{-1} \mathrm{~K}^{-1 \mathrm{a}}$ \\
$T_{2}$, & $38^{\circ} \mathrm{C}$ \\
$C$, & $6.6 \mathrm{kcal} \mathrm{mol}^{-1}$ \\
$\Delta \mu$, & $9.5 \mathrm{kcal} \mathrm{mol}^{-1}$ \\
$S_{\mathrm{e}}\left(T_{\mathrm{g}^{0}}, 0\right)$, & $0.00767 \mathrm{cal} \mathrm{g}^{-1} \mathrm{~K}^{-1}$ \\
\hline
\end{tabular}

a Wunderlich's data.

the sample calculated from $a_{2} . T_{2}$ is nearly equal to $T_{\mathrm{g}}-40\left({ }^{\circ} \mathrm{C}\right)$, which is a reasonable relation. Since $S_{\mathrm{e}}\left(T_{2}, 0\right)=0$, the configurational entropy at $T_{\mathrm{g}}{ }^{0}$ under atmospheric pressure is given by $S_{\mathrm{e}}\left(T_{\mathrm{g}}{ }^{0}, 0\right)=\Delta C_{\mathrm{P}} \ln \left(T_{\mathrm{g}}{ }^{0} / T_{2}\right)$. Thus the value of $S_{\mathrm{e}}\left(T_{\mathrm{g}}{ }^{0}, 0\right)$ can be obtained from the parameter $a_{2}$ (Table I). Consequently $S_{\mathrm{e}}$ at an arbitrary temperature and pressure for present $\mathrm{PVC}$ is given as

$$
\begin{aligned}
S_{\mathrm{e}}(T, P)= & 0.00767+0.161 \log \left(T / T_{\mathrm{g}}{ }^{0}\right) \\
& -\int_{0}^{P} V \Delta \alpha \mathrm{d} P\left[\mathrm{cal} \mathrm{g}^{-1} \mathrm{~K}^{-1}\right]
\end{aligned}
$$

The FS-type relation can be derived from the Adam-Gibbs theory, if we assume that $S_{\mathrm{e}}$ is written by the following relation,

$$
\begin{aligned}
S_{\mathrm{e}}(T, P)= & S_{\mathrm{e}}\left(T_{\mathrm{g}}{ }^{0}, 0\right)+\Delta C_{\mathrm{P}} \ln \left(T / T_{\mathrm{g}}{ }^{0}\right) \\
& -\int_{0}^{P} V \Delta \alpha \mathrm{d} P=S_{\mathrm{e} 0}-\overline{V \Delta \alpha} P
\end{aligned}
$$

where $S_{\mathrm{e} 0}$ is the excess entropy at atmospheric pressure and at temperature $T\left(>T_{\mathrm{g}}{ }^{0}\right)$, and $\Delta \alpha$ denotes difference in the expansion coefficient between the equilibrium melt and the glass, respectively. Since $V$ and $\Delta \alpha$ at temperature $T$ are functions of pressure, their average values within the measured pressure range are designated as $\bar{V}$ and $\overline{\Delta \alpha}$. As a result of simple mathematical treatment, it is easily found that FS parameters $b_{1}$ and $b_{2}$ can be given by $b_{1}=s_{\mathrm{c}} * \Delta \mu / 2.3 k T S_{\mathrm{e} 0}$ and $b_{2}=S_{\mathrm{e} 0} / \overline{V \Delta \alpha}$.

\section{Criterion for the Glass Tansition}

Glass-transition temperature at atmospheric pressure, $T_{\mathrm{g}}{ }^{0}$, is generally defined by the breaking point of the volume-temperature curve above which a liquid-like expansion exists and below which a glass-like expansion exists. Since the dilatometric measurement under high pressure was impossible in our experiments, a glasstransition temperature at a pressure $P, T_{\mathrm{g}}{ }^{\mathrm{P}}$, must be estimated from other pertinent means. As reported in another paper, ${ }^{7}$ d.c. conduction in polymers is supposed to be due to ionic impurities. The mobility of charge carriers changes remarkably at the point where the host polymer is expected in the temperature and pressure dependences of the conductivity. In practice, $\log \sigma$ vs. $T$ plot at $1 \mathrm{~atm}$. has a breaking point at $T=T_{\mathrm{b}}$, and $T_{\mathrm{b}}$ corresponds to $T_{\mathrm{g}}{ }^{0}$ defined from the volume-temperature relation. Here $\sigma$ is the d.c. conductivity of the polymer. As shown in Figure 2, the curve of $\log \sigma$ vs. $P$ at a fixed temperature also has a breaking point at $\boldsymbol{P}=\boldsymbol{P}_{\mathrm{b}}$ (indicated by an arrow), which is believed to correspond to the glass transition pressure at the temperature. Therefore, $T_{\mathrm{g}}{ }^{\mathrm{P}}$ was determined from the breaking point of $\log \sigma v s$. $P$ curve here, though actual specification of $T_{\mathrm{g}}{ }^{0}$ 
depends upon what time scale has been used to define $T_{\mathrm{g}}{ }^{0}$.

Figure 6 shows the pressure dependence of $T_{\mathrm{g}}{ }^{\mathrm{P}}$ for PVC. $T_{\mathrm{g}}{ }^{\mathrm{P}}$ changes with $\boldsymbol{P}$ nearly in a

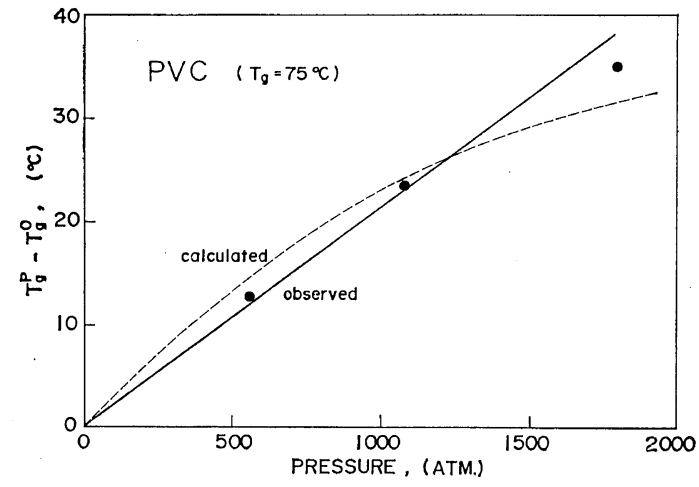

Figure 6. Change of glass-transition temperature $T_{\mathrm{g}}{ }^{\mathrm{P}}$ with pressure.

linear fashion, and the temperature-pressure coefficient, $\mathrm{d} T_{\mathrm{g}} / \mathrm{d} P$, is equal to $2.15 \times 10^{-2} \mathrm{~K}$ atm. ${ }^{-1}$. This is considerably larger than the value $1.35 \times 10^{-2} \mathrm{~K} \mathrm{~atm} .^{-1}$ obtained by Hellwege, et al. ${ }^{8}$ from dilatometric measurements.

If the glass transition is approximated by freezing of a single internal parameter such as excess entropy and excess enthalpy, it follows that

$$
\frac{\mathrm{d} T_{\mathrm{g}}}{\mathrm{d} P}=\frac{T_{\mathrm{g}} V \Delta \alpha}{\Delta C_{\mathrm{P}}}
$$

It has been concluded by Goldstein ${ }^{2}$ and by $\mathrm{Gee}^{9}$ on the basis of experimental data available that eq 4 holds approximately. This fact indicates that the glass transition is the freezing process of two or more internal variables, but, if one variable is to be adopted for describing the glass transition, the excess entropy or excess enthalpy is a better one than the excess volume. Integrating eq 4 with respect to $P$,

$$
\frac{T_{\mathrm{g}}^{\mathrm{P}}-T_{\mathrm{g}}{ }^{0}}{T_{\mathrm{g}}{ }^{0}} \simeq \frac{1}{\Delta C_{\mathrm{P}}} \int_{0}^{P \mathrm{~g}} V \Delta \alpha \mathrm{d} P
$$

Here it is assumed that changes of $\Delta C_{\mathrm{P}}$ with $T$ and $P$ are negligible in the temperature and pressure ranges available. Hence $T_{\mathrm{g}}{ }^{\mathrm{P}}$ can be numerically calculated in the same procedure as $S_{\mathrm{e}}{ }^{\prime}$ (see Appendix). This result is shown in

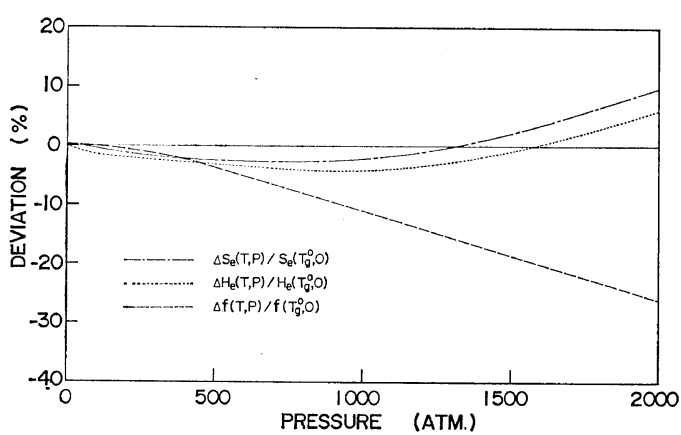

Figure 7. Effect of pressure on excess quantities, $\Delta S_{\mathrm{e}}, \Delta H_{\mathrm{e}}$, and $\Delta f$.

Figure 6 by a broken line.

Subsequently attention can be focused on the problem as to how large are the values of $\Delta S_{\mathrm{e}}$, $\Delta H_{\mathrm{e}}$, and $\Delta f$ at the glass transition. Figure 7 shows effects of pressure on the excess quantities $\Delta S_{\mathrm{e}}, \Delta H_{\mathrm{e}}$, and $\Delta f$ at $T_{\mathrm{g}}{ }^{\mathrm{P}}$. Values of these excess quantities at $T_{\mathrm{g}}{ }^{\mathrm{P}}$ were determined from Figures $\mathrm{A} 1 \sim \mathrm{A} 3$. In order to compare the relative magnitude among these excess quantities, the ordinate in Figure 7 indicates the variations of them relative to values of the quantities at $T_{\mathrm{g}}{ }^{0}$. $f\left(T_{\mathrm{g}}{ }^{0}, 0\right)$ was estimated from the WLF parameter $C_{1}$ and found to be $0.027 . S_{\mathrm{e}}\left(T_{\mathrm{g}}{ }^{0}, 0\right)$ was estimated as $0.00767 \mathrm{cal} \mathrm{g}^{-1} \mathrm{~K}^{-1}$ from the AdamGibbs theory. At $T=T_{\mathrm{g}}{ }^{0}, H_{\mathrm{e}}\left(T_{\mathrm{g}}{ }^{0}, 0\right)$ may be approximately equal to $T_{\mathrm{g}}{ }^{0} S_{\mathrm{e}}\left(T_{\mathrm{g}}{ }^{0}, 0\right)$. Then $H_{\mathrm{e}}\left(T_{\mathrm{g}}{ }^{0}, 0\right)$ was estimated as $2.67 \mathrm{cal} \mathrm{g}^{-1}$. If a quantity $X(T, P)$ is the determining factor of the glass transition, the excess quantity $\Delta X=X\left(T_{\mathrm{g}}{ }^{\mathrm{P}}, P\right)-X\left(T_{\mathrm{g}}{ }^{0}, 0\right)$ should be independent of $P$ and equal to zero; its variation with pressure should coincide with the zero level in Figure 7. As shown in Figure 7, $\Delta f$ dęviates considerably from 0 with increasing pressure, while $\Delta S_{\mathrm{e}}$ or $\Delta H_{\mathrm{e}}$ deviates slightly. Therefore, $S_{\mathrm{e}}$ or $H_{\mathrm{e}}$ seems to be much better for the determining factor of the glass transition than $f$; that is, the glass transition would be observed at the time scale of the measurements when $S_{\mathrm{e}}$ or $H_{\mathrm{e}}$ reaches a certain value.

The relaxation time $\tau$ at $T_{\mathrm{g}}{ }^{\mathrm{P}}, \tau_{\mathrm{g}}{ }^{\mathrm{P}}$, can be determined from the FS equation (eq 2) and $\boldsymbol{P}_{\mathrm{g}}$. Values of $\tau_{\mathrm{g}}{ }^{\mathrm{P}}$ are listed in Table II. It is very curious that $\tau_{\mathrm{g}}{ }^{\mathrm{P}}$ decreases exponentially with increase in $T_{\mathrm{g}}{ }^{\mathrm{P}}$. According to the phenomenological definition of $T_{\mathrm{g}}, \tau_{\mathrm{g}}$ should be of 
Table II. Values of FS parameters $b_{1}$ and $b_{2}$, and $\tau_{\mathrm{g}}{ }^{P}$

\begin{tabular}{rrrrc}
\hline$T^{\circ} \mathrm{C}$ & $P_{\mathrm{g}}$, atm. & $b_{1}$ & $b_{2}$, atm. & $\log \tau_{\mathrm{g}}{ }^{P}$ \\
\hline 75 & 0 & - & - & 2.5 \\
98 & 1070 & 14.3 & 5084 & -0.25 \\
107 & 1490 & 6.8 & 4245 & -1.46 \\
121 & 2140 & 12.8 & 8358 & -1.95 \\
130 & 2560 & 11.8 & 10290 & -3.18 \\
\hline
\end{tabular}

the same order of magnitude as the experimental time scale independently of pressure and the nature of the sample. If we assume, however, the model that the segmental motion is a redistribution process of vacant sites in the configurational space of the polymer and that the temperature rise causes the increase in the number of excited states in the potential field formed by surrounding molecules for a diffusive displacement of a segmental unit and the pressure increase causes the change of the curvature of potential field, this behavior might be explained; the number of rearrangeable vacant sites is reduced by applying external pressure $P$ (that is, the reduction of excess entropy) but the rate of rearranging of segmental units is not so affected by $P$, since the latter is mainly determined by $T$. Therefore at $T_{\mathrm{g}}{ }^{\mathrm{P}}\left(>T_{\mathrm{g}}{ }^{0}\right), \tau_{\mathrm{g}}{ }^{\mathrm{P}}$ is shorter than $\tau_{\mathrm{g}}{ }^{0}$.

\section{CONCLUSION}

The relaxation time for the $\alpha_{\mathrm{a}}$ relaxation process which is concerned with the rate of the segmental motion depends on temperature and pressure following the WLF and FS equations, respectively. The relaxation time is mainly controlled by the excess entropy $S_{\mathrm{e}}$ and/or excess enthalpy $H_{\mathrm{e}}$ rather than by the excess volume fraction $f$. If we assume the applicability of the Adam-Gibbs theory to the temperature dependence of the relaxation time, the excess transition) can be obtained numerically for the present PVC sample.

The glass-transition point can be determined from the break point of the logarithm of the d.c. conductivity vs. temperature or pressure, and then the temperature-pressure coefficient of the glass transition, $\mathrm{d} T_{\mathrm{g}} / \mathrm{d} P$, is easily deentropy at arbitrary $T$ and $P$ (above the glass

termined. For the present sample, $\mathrm{d} T_{\mathrm{g}} / \mathrm{d} \boldsymbol{P}$ was found to be $2.15 \times 10^{-2} \mathrm{Katm}^{-1}$. It might be concluded that the determining factor of the glass transition is the excess entropy and/or excess volume fraction. The relaxation time at $T_{\mathrm{g}}{ }^{\mathrm{P}}, \tau_{\mathrm{g}}{ }^{\mathrm{P}}$, decreases exponentially with increase in $T_{\mathrm{g}}{ }^{\mathrm{P}}$, but the reason for this is open to speculation as yet.

\section{APPENDIX}

Calculation of Excess Entropy, Excess Enthalpy, and Excess Volume

We will show the procedure of derivation of thermodynamic variables, entropy $S$, and enthalpy $H$, from experimentally observable quantities. Since $S$ and $H$ are functions of two independent variables of temperature $T$ and pressure $P$, infinitesimal changes in $S$ and $H$ are given as

$$
\begin{aligned}
\mathrm{d} S & =(\partial S / \partial T)_{\mathrm{P}} \mathrm{d} T+(\partial S / \partial P)_{\mathrm{T}} \mathrm{d} P \\
& =\left(C_{\mathrm{P}} / T\right) \mathrm{d} T+(-V \alpha) \mathrm{d} P \\
\mathrm{~d} H & =(\partial H / \partial T)_{\mathrm{P}} \mathrm{d} T+(\partial H / \partial P)_{\mathrm{T}} \mathrm{d} P \\
& =C_{\mathrm{P}} \mathrm{d} T+(V-T V \alpha) \mathrm{d} P
\end{aligned}
$$

where $\alpha$ is the thermal expansion coefficient and $C_{\mathrm{P}}$ is the specific heat at constant pressure. With the assumption of additivity in $S$, an excess (or a configurational) entropy $S_{\mathrm{e}}$ is defined by the difference between $S$ in the liquid state, $S_{\mathrm{L}}$, and that in the hypothetical glassy state, $S_{\mathrm{G}}$. An excess (or a configurational) enthalpy $H_{\mathrm{e}}$ is also defined as $H_{\mathrm{e}}=H_{\mathrm{L}}-H_{\mathrm{G}}$. Then changes in these excess quantities near the glass-transition temperature $T_{\mathrm{g}}{ }^{\mathrm{P}}$ under external pressure $\boldsymbol{P}$ are given as

$$
\begin{aligned}
\mathrm{d} S_{\mathrm{e}}= & \mathrm{d} S_{\mathrm{L}}-\mathrm{d} S_{\mathrm{G}} \simeq\left(\Delta C_{\mathrm{P}} / T\right) \mathrm{d} T-V\left(T_{\mathrm{g}}{ }^{0}, 0\right) \Delta \alpha \mathrm{d} P \\
\mathrm{~d} H_{\mathrm{e}}= & \mathrm{d} H_{\mathrm{L}}-\mathrm{d} H_{\mathrm{G}} \simeq \Delta C_{\mathrm{P}} \mathrm{d} T+\Delta V \mathrm{~d} P \\
& -T V\left(T_{\mathrm{g}}{ }^{0}, 0\right) \Delta \alpha \mathrm{d} P
\end{aligned}
$$

where $\Delta \alpha=\alpha_{\mathrm{L}}-\alpha_{\mathrm{G}}, \Delta V=V_{\mathrm{L}}-V_{\mathrm{G}}$, and suffices $\mathrm{L}$ and $\mathrm{G}$ indicate the liquid state and glassy state, respectively. $\Delta C_{\mathrm{P}}$ is the discontinuity in $C_{\mathrm{P}}$ at $T_{\mathrm{g}}{ }^{\mathrm{P}}$. These equations are written in the integral forms:

$$
\int_{\left(T_{\mathrm{g}}{ }^{0}, 0\right)}^{(T, P)} \mathrm{d} S_{\mathrm{e}}=\int_{T_{\mathrm{g}^{0}}}^{T}\left(\Delta C_{\mathrm{P}} / T\right) \mathrm{d} T-\int_{0}^{P} V \Delta \alpha \mathrm{d} P
$$




$$
\begin{aligned}
\int_{\left(\mathrm{g}_{\mathrm{g}}, 0\right)}^{(T, P)} \mathrm{d} H_{\mathrm{e}}= & \int_{T_{\mathrm{g}^{0}}}^{T} \Delta C_{\mathrm{P}} \mathrm{d} T+\int_{0}^{P} \Delta V \mathrm{~d} P \\
& -\int_{0}^{P} T V \Delta \alpha \mathrm{d} P
\end{aligned}
$$

Here the glass-transition temperature at atmospheric pressure $T_{\mathrm{g}}{ }^{0}$ is regarded as the reference state. Although $\Delta C_{\mathrm{P}}$ is slightly dependent on $T$ and $P$, we assume for simplicity that $\Delta C_{\mathrm{P}}$ is independent of them. Then, $S_{\mathrm{e}}$ and $H_{\mathrm{e}}$ are obtained as

$$
\begin{aligned}
S_{\mathrm{e}}(T, P)= & S_{\mathrm{e}}\left(T_{\mathrm{g}}{ }^{0}, 0\right)+\Delta C_{\mathrm{P}} \ln \left(T / T_{\mathrm{g}}{ }^{0}\right) \\
& -\int_{0}^{P} V \Delta \alpha \mathrm{d} P \\
H_{\mathrm{e}}(T, P)= & H_{\mathrm{e}}\left(T_{\mathrm{g}}{ }^{0}, 0\right)+\Delta C_{\mathrm{P}}\left(T-T_{\mathrm{g}}{ }^{0}\right) \\
& +\int_{0}^{P} \Delta V \mathrm{~d} P-T \int_{0}^{P} V \Delta \alpha \mathrm{d} P
\end{aligned}
$$

Experimental isotherms at elevated pressures for liquids are well described by the empirical Tait equation,

$$
V=V_{0}[1-C \ln (1+P / B)]
$$

where $V_{0}$ represents the initial volume at atmospheric pressure $(\boldsymbol{P} \simeq 0)$, and $C$ and $B$ are constants. Tait equation would be also a good expression for the compression of linear polymers in the liquid state. ${ }^{10,11}$ The parameter $C$ is independent of $T$ and characteristics of polymers, and has a universal value of 0.0894 for linear polymers. ${ }^{11}$ The parameter $B$, on the contrary, depends on temperature and the specific system. Differentiating eq A7 with respect to $T$ and $P$, we get the thermal expansion coefficient $\alpha$ and the isothermal compressibility $\beta$, respectively;

$$
\begin{aligned}
& \alpha(P) \equiv(\partial \ln V / \partial T)_{\mathrm{P}}=\alpha_{0}+P \beta(\partial \ln B / \partial T) \\
& \beta(P) \equiv-(\partial \ln V / \partial P)_{\mathrm{T}}=V_{0} C / V(P+B)
\end{aligned}
$$

For simplicity, we assume that the Tait equation can be also applied to polymers in the glassy state, then $\alpha$ and $\beta$ for polymers below $T_{\mathrm{g}}{ }^{\mathrm{P}}$ are described with eq A8 and A9, respectively.

In order to obtain numerical values of $S_{\mathrm{e}}$ and $H_{\mathrm{e}}$ as a function of temperature and pressure, we need volumetric and calorimetric data of the polymers. Unfortunately, volumetric measurements under high pressure were impossible at this stage of our experiments. Therefore we must estimate values of volume at arbitrary temperature and pressure with proper means. Hellwege, Knappe, and Lehmann ${ }^{8}$ studied isothermal compression of five polymers. Let's introduce their data on PVC to our analysis. Applying eq A7 in the form,

$$
\left(-V_{0} / \Delta V\right) C \Delta P=B+\bar{P}
$$

to the data, parameters $B$ and $C$ are easily determined from the intercept and the slope of a linear plot of $\left(-V_{0} / \Delta V\right) \Delta P$ vs. $\bar{P}$ as a function of temperature, respectively. Here $\bar{P}$ and $\Delta V$ are an average pressure and a volume change in the range of $P \sim P+\Delta P$, respectively, and $\Delta P$ is a pressure interval. $C$ is nearly constant $(\simeq 0.09)$ in the liquid and the glassy states, $B_{\mathrm{L}}$ is determined from the liquid state line below the glass transition pressure $\boldsymbol{P}_{\mathrm{g}}$, and $\boldsymbol{B}_{\mathrm{G}}$ from the glassy state line above $\boldsymbol{P}_{\mathrm{g}}$. As Gee pointed out in his analysis on the compression of polystyrene, ${ }^{9}$ $\ln B_{\mathrm{L}}$ and $\ln B_{\mathrm{G}}$ for the present PVC are both linear function of $T$, and given as

$$
\log B_{\mathrm{L}}=4.4635-3.10 \times 10^{-3} T
$$

and $\quad \log B_{G}=3.8774-1.10 \times 10^{-3} T$

where $T$ is an absolute temperature. Consequently, values of a liquid volume $V_{\mathrm{L}}$ and a glass volume $V_{G}$ at $T$ and $P$ can be obtained from the Tait equation (eq A7). We introduce a value of $\Delta C_{\mathrm{P}}$ obtained by Wunderlich ${ }^{12}$ $\left(\Delta C_{\mathrm{P}}=4.26 \mathrm{kcal} \mathrm{mol}^{-1} \mathrm{~K}^{-1}=0.068 \mathrm{cal} \mathrm{g}^{-1} \mathrm{~K}^{-1}\right)$.

As can be seen from eq $\mathrm{A} 5$ and A6, pressure dependences of $S_{\mathrm{e}}$ and $H_{\mathrm{e}}$ are represented in terms of the integrals $\int_{0}^{P} V \Delta \alpha \mathrm{d} P$ and $\int_{0}^{P} \Delta V \mathrm{~d} P$. These integrals are carried out graphically; that is, the areas under the curves of $V \Delta \alpha$ vs. $P$ and $\Delta V$ vs. $P$ plots over the range from 0 to $P$ atm. are calculated on the graph. The value of $\Delta \alpha$ at $\boldsymbol{P}$ is given from eq $\mathrm{A} 8$ as

$\Delta \alpha(\boldsymbol{P})=\Delta \alpha(0)+P\left[\beta_{\mathrm{L}}\left(\partial \ln \boldsymbol{B}_{\mathrm{L}} / \partial T\right)-\beta_{\mathrm{G}}\left(\partial \ln \boldsymbol{B}_{\mathrm{G}} / \partial T\right)\right]$

where $\Delta \alpha(0)$ is the discontnuity in $\alpha$ at $T_{\mathrm{g}}{ }^{0} \quad(75$ ${ }^{\circ} \mathrm{C}$ ) and estimated from the volume-temperature curve as $3.35 \times 10^{-4} \mathrm{~K}^{-1}$. Hence excess quantities $\Delta S_{\mathrm{e}}$ and $\Delta H_{\mathrm{e}}$ are given as 


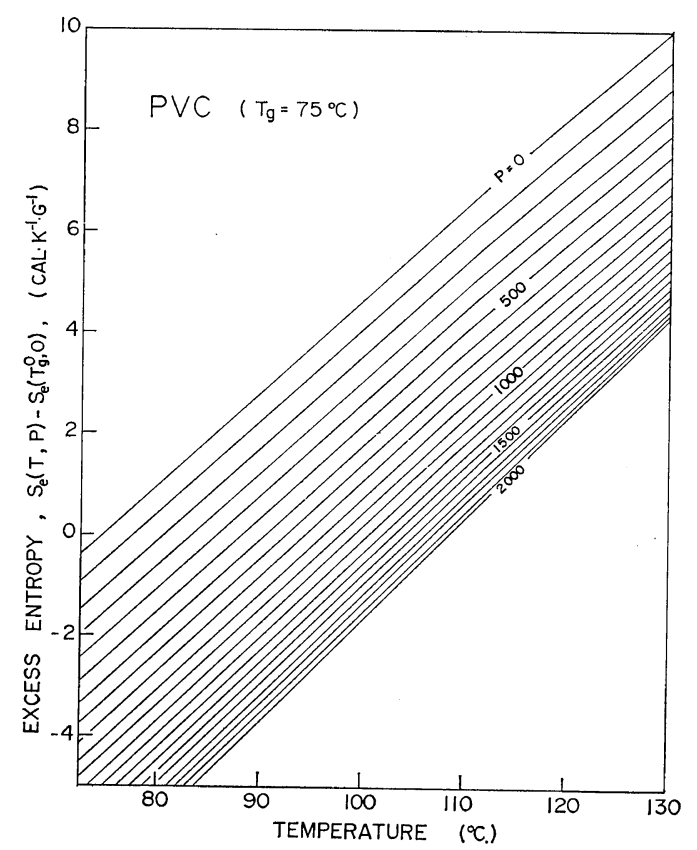

Figure A1. Temperature and pressure dependences of excess entropy $\Delta S_{\mathrm{e}}$.

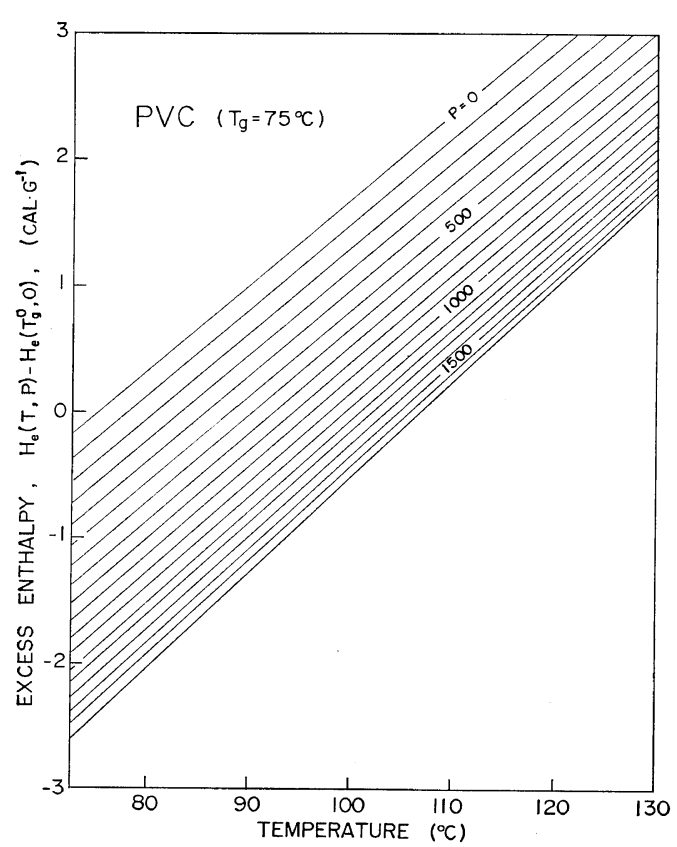

Figure A2. Temperature and pressure dependences of excess enthalpy $\Delta H_{\mathrm{e}}$.

$$
\begin{aligned}
\Delta S_{\mathrm{e}}(T, P) & =S_{\mathrm{e}}(T, P)-S_{\mathrm{e}}\left(T_{\mathrm{g}}{ }^{0}, 0\right) \\
& =\Delta C_{\mathrm{P}} \ln \left(T / T_{\mathrm{g}}{ }^{0}\right)-\int_{0}^{P} V \Delta \alpha \mathrm{d} P \quad(\mathrm{~A} 13) \\
\Delta H_{\mathrm{e}}(T, P) & =H_{\mathrm{e}}(T, P)-H_{\mathrm{e}}\left(T_{\mathrm{g}}{ }^{0}, 0\right) \\
& =\Delta C_{\mathrm{P}}\left(T-T_{\mathrm{g}}{ }^{0}\right)+\int_{0}^{P} \Delta V \mathrm{~d} P-T \int_{0}^{P} V \Delta \alpha \mathrm{d} P
\end{aligned}
$$

If we assume that the excess volume in liquid state or in glassy state is defined as the difference between crystal volume and liquid volume $V_{\mathrm{L}}$ or glass volume $V_{\mathrm{G}}$ and that the thermal expansion coefficient and isothermal compression of the crystal and equal to those of the glass, we can define the free volume as the free volume at $T_{\mathrm{g}}$ plus the excess volume from the linear extrapolation of glass volume. Then the fractional free volume $f(T, P)$ defined as the ratio of free volume to volume at $T_{\mathrm{g}}{ }^{\mathrm{P}}$ is expressed as

$$
\begin{aligned}
& f(T, P)= \\
& \quad\left[V_{\mathrm{L}}(T, P)-V_{\mathrm{G}}(T, P)\right] / V_{\mathrm{L}}\left(T_{\mathrm{g}}{ }^{\mathrm{P}}, P\right)+f\left(T_{\mathrm{g}}{ }^{0}, 0\right)
\end{aligned}
$$

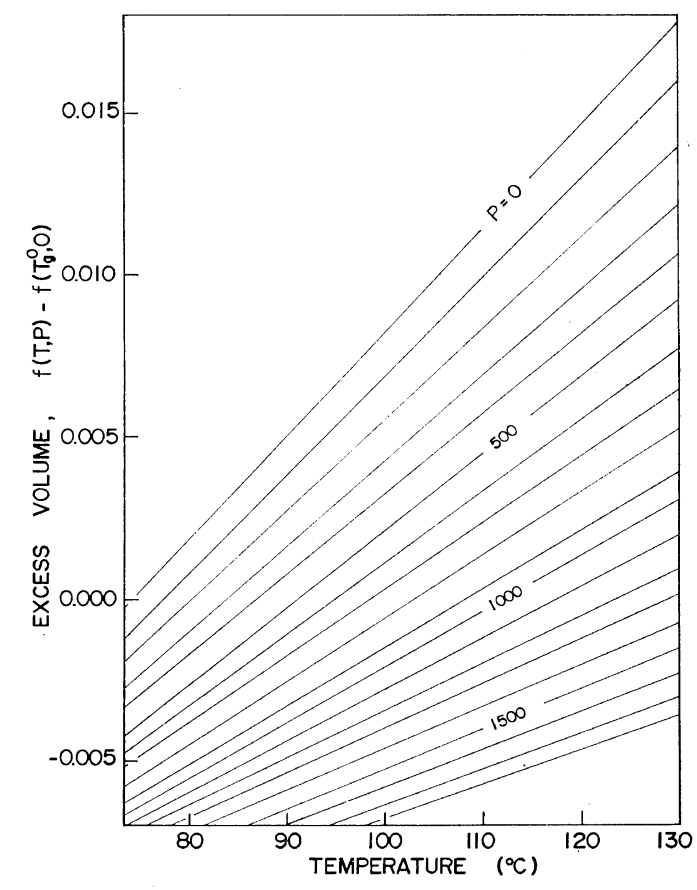

Figure A3. Temperature and pressure dependences of excess volume $\Delta f$. 
and hence the excess volume fraction $\Delta f$ is given as

$$
\begin{aligned}
\Delta f(T, P) & =f(T, P)-f\left(T_{\mathrm{g}}{ }^{0}, 0\right) \\
& =\left[V_{\mathrm{L}}(T, P)-V_{\mathrm{G}}(T, P)\right] / V_{\mathrm{L}}\left(T_{\mathrm{g}}{ }^{\mathrm{P}}, P\right)
\end{aligned}
$$

Numerical results of excess quantities, $\Delta S_{\mathrm{e}}$, $\Delta H_{\mathrm{e}}$, and $\Delta f$, for PVC are respectively shown in Figures $\mathrm{A} 1 \sim \mathrm{A} 3$ as a function of $T$ and $P$.

\section{REFERNCES}

1. N. I. Shishkin, Soviet Phys. Soiid State, 2, 322 (1960).

2. M. Goldstein, J. Chem. Phys., 39, 3369 (1963).

3. M. L. Williams, R. F. Landel, and J. D. Ferry, J. Amer. Chem. Soc., 77, 3701 (1955).
4. J. D. Ferry and R. A. Stratton, Kolloid Z., 171, 107 (1960).

5. S. Saito, H. Sasabe, T. Nakajima, and K. Yada, J. Polym. Sci., Part A-2, 6, 1297 (1968).

6. G. Adam and J. H. Gibbs, J. Chem. Phys., 45, 139 (1965).

7. S. Saito, to be submitted to J. Polym. Sci. Part. $A-2$.

8. K. H. Hellwege, W. Knappe, and P. Lehmann, Kolloid Z. Z. Polym. 183, 110 (1962).

9. G. Gee, Polymer, 7, 177 (1966).

10. W. G. Cutler, R. H. McMickel, W. Webb, and R. W. Sciessler, J. Chem. Phys., 29, 727 (1958).

11. V. S. Nanda and R. Simha, J. Chem. Phys., 41, 1884 (1964).

12. B. Wunderlich, J. Phys. Chem., 64, 1052 (1960). 